\title{
REMARKS ON WEAK COMPACTNESS OF OPERATORS DEFINED ON CERTAIN INJECTIVE TENSOR PRODUCTS
}

\author{
G. EMMANUELE
}

(Communicated by Palle E. T. Jorgensen)

\begin{abstract}
We show that if $X$ is a $\mathscr{L}_{\infty}$-space with the Dieudonne property and $Y$ is a Banach space not containing $l_{1}$, then any operator $T: X \otimes_{\varepsilon} Y \rightarrow Z$, where $Z$ is a weakly sequentially complete Banach space, is weakly compact. Some other results of the same kind are obtained.
\end{abstract}

Let $X$ be a $\mathscr{L}_{\infty}$-space (see [1] for this notion and some useful results on $\mathscr{L}_{\infty}$ spaces) and $Y$ be a Banach space not containing $l_{1}$. We consider the injective tensor product $X \otimes_{\varepsilon} Y$ (see [3]), and we investigate the following problem: when is any operator $T: X \otimes_{\mathcal{E}} Y \rightarrow Z$, where $Z$ is a Banach space, weakly compact?

In the case of $X=C(K)$ there are some papers devoted to the study of this question (see [2, 6-9]), but nothing seems to be known in the present setting; we observe that the theorems proved in the paper extend all of the above-quoted results, but their proofs make use of the results in [2, 8], so that they may be considered interesting complements to those theorems. Because the proofs of our results are similar, we give the proof of Theorem 2 only and leave the others to the reader. We need the following definition: a Banach space $E$ has the Dieudonné property if any weakly completely continuous (or Dieudonné) operator defined on it is weakly compact [8].

We recall that $C(K)$ spaces have the Dieudonne property.

Lemma 1. $X^{* *} \otimes_{\varepsilon} Y$ is a closed subspace of $\left(X \otimes_{\varepsilon} Y\right)^{* *}$.

Proof. Let $x^{* *} \otimes y$ be an element of $X \otimes Y$ and consider $\varphi \in\left(X \otimes_{\varepsilon} Y\right)^{*}=$ $B^{\pi}(X, Y)$ (see [3] for a definition of $B^{\pi}(X, Y)$ ). Since $B^{\pi}(X, Y)$ is a closed subspace of $B^{\pi}\left(X^{* *}, Y\right)=\left(X^{* *} \otimes_{\varepsilon} Y\right)^{*}$ (see [3]) and since $\|\varphi\|_{B^{\pi}(X, Y)}=$ $\|\varphi\|_{B^{\pi}\left(X^{* *}, Y\right)}$ for any $\varphi \in B^{\pi}(X, Y)$, we have $\left|\varphi\left(x^{* *} \otimes y\right)\right| \leq\|\varphi\|_{\pi}\left\|x^{* *} \otimes y\right\|_{\varepsilon}$ and so $x^{* *} \otimes y \in\left(X \otimes_{\varepsilon} Y\right)^{* *}$; hence $\sum_{i=1}^{p} x_{i}^{* *} \otimes y_{i} \in\left(X \otimes_{\varepsilon} Y\right)^{* *}$. Now, we have to show that $\left\|\sum_{i=1}^{p} x_{i}^{* *} \otimes y_{i}\right\|_{\varepsilon}=\left\|\sum_{i=1}^{p} x_{i}^{* *} \otimes y_{i}\right\|_{\left(X \otimes_{\varepsilon} Y\right)^{* *}}$. The inequality $\left\|\sum_{i=1}^{p} x_{i}^{* *} \otimes y_{i}\right\|_{\varepsilon} \leq\left\|\sum_{i=1}^{p} x_{i}^{* *} \otimes y_{i}\right\|_{\left(X \otimes_{\varepsilon} Y\right)^{* *} \text { follows very easily from the very }}$ definition of $\varepsilon$-norm [3], the weak ${ }^{*}$ density of $B_{X^{*}}$ in $B_{X^{* \cdots}}$, and the fact that

Received by the editors March 22, 1991.

1991 Mathematics Subject Classification. Primary 47B99, 46M05.

Key words and phrases. Weak compactness, injective tensor products.

Work performed under the auspices of G.N.A.F.A. of C.N.R. and partially supported by M.U.R.S.T. of Italy (40\%). 
any $x^{*} \otimes y^{*} \in B^{\pi}(X, Y)$. The reverse inequality follows since we have

$$
\left\|\sum_{i=1}^{p} x_{i}^{* *} \otimes y_{i}\right\|_{\left(X \otimes_{\ell} Y\right)^{* *}}=\sup \left\{\left|\left(\sum_{i=1}^{p} x_{i}^{* *} \otimes y_{i}\right)(\varphi)\right|: \varphi \in B^{\pi}(X, Y),\|\varphi\|_{\pi} \leq 1\right\},
$$

any $\varphi \in B^{\pi}(X, Y)$ actually is an element of $B^{\pi}\left(X^{* *}, Y\right)$, and this inclusion is an isometry [3], whereas we also have

$$
\left\|\sum_{i=1}^{p} x_{i}^{* *} \otimes y_{i}\right\|_{\varepsilon}=\sup \left\{\left|\left(\sum_{i=1}^{p} x_{i}^{* *} \otimes y_{i}\right)(\varphi)\right|: \varphi \in B^{\pi}\left(X^{* *}, Y\right),\|\varphi\|_{\pi} \leq 1\right\} .
$$

Because the elements of the type $\sum_{i=1}^{p} x_{i}^{* *} \otimes y_{i}$ are dense in $X^{* *} \otimes_{\varepsilon} Y$, we are done.

We are now ready for the first theorem of the paper.

Theorem 2. Let $X$ be a $\mathscr{L}_{\infty}$-space with the Dieudonné property and $Y$ be a Banach space not containing $l_{1}$. If $Z$ is a weakly sequentially complete Banach space, then any $T: X \otimes_{\varepsilon} Y \rightarrow Z$ is weakly compact.

Proof. Let us consider $T^{* *}:\left(X \otimes_{\varepsilon} Y\right)^{* *} \rightarrow Z^{* *}$ and its restriction $\tilde{T}$ to all of $X^{* *} \otimes_{\varepsilon} Y$ (which contains $X \otimes_{\varepsilon} Y$ as a closed subspace [3]). $\tilde{T}$ is continuous, because $X^{* *} \otimes_{\varepsilon} Y$ is a closed subspace of $\left(X \otimes_{\varepsilon} Y\right)^{* *}$, by virtue of Lemma 1 .

We want to prove that $\widetilde{T}$ takes its values in $Z$. Consider $x^{* *} \otimes y \in B_{x^{* *} \otimes_{\varepsilon} Y}$ and suppose (as we may) that $\left\|x^{* *}\right\| \leq 1$. Then there is a net $\left(x_{\alpha}\right) \subset B_{x}$ such that $x_{\alpha} \rightarrow^{w^{*}} x^{* *}$. Of course, we have

$$
w^{*}-\lim _{\alpha} \tilde{T}\left(x_{\alpha} \otimes y\right)=\widetilde{T}\left(x^{* *} \otimes y\right) .
$$

Now define $\widetilde{T}_{y}: X \rightarrow Z$ by putting $\widetilde{T}_{y}(x)=T(x \otimes y) . \widetilde{T}_{y}$ is a Dieudonné operator since $Z$ is weakly sequentially complete; and so $\widetilde{T}_{y}$ is weakly compact, since $X$ has the Dieudonne property. Hence $\left(\widetilde{T}_{y}\left(x_{\alpha}\right)\right)$ is a relatively weakly compact subset of $Z$; because $\widetilde{T}_{y}\left(x_{\alpha}\right)=T\left(x_{\alpha} \otimes y\right)=\widetilde{T}\left(x_{\alpha} \otimes y\right)$ for all $\alpha$, the weak closure of the net $\widetilde{T}\left(x_{\alpha} \otimes y\right)$ is in $Z$ and so even any weak * cluster point of $\tilde{T}\left(x_{\alpha} \otimes y\right)$ must lie in $Z$; hence $\widetilde{T}\left(x^{* *} \otimes y\right) \in Z$. This means that $\widetilde{T}\left(\sum_{i=1}^{p} x_{i}^{* *} \otimes y_{i}\right) \in Z$ for any $\sum_{i=1}^{p} x_{i}^{* *} \otimes y_{i} \in X^{* *} \otimes Y$. The density of the elements of the type $\sum_{i=1}^{p} x_{i}^{* *} \otimes y_{i}$ in $X^{* *} \otimes_{\varepsilon} Y$ and the continuity of $\tilde{T}$ on $X^{* *} \otimes_{\varepsilon} Y$ give our claim: $\widetilde{T}$ takes its values in $Z$.

Now recall that $X^{* *}$ has the metric approximation property (see [3]) and so $X^{* *} \otimes_{\varepsilon} Y=K_{w^{*}}\left(Y^{*}, X^{* *}\right) \quad\left(=\right.$ the Banach space of all $w^{*}-w$ continuous compact operators from $Y^{*}$ into $\left.X^{* *}\right)$; furthermore, $X^{* *}$ is complemented in some $C(K)$ space [1] and so $K_{w^{*}}\left(Y^{*}, X^{* *}\right)$ is complemented in $C(K, Y)$ by a projection $P$. The operator $\widetilde{T} \circ P: C(K, Y) \rightarrow Z$ is a Dieudonné operator that must be weakly compact because of the result of [8]. Since $\widetilde{T} \circ P$ restricted to $X \otimes_{\varepsilon} Y$ is nothing else than $T$, we are done.

A similar proof (making use of the main result of the paper [2]) gives the following theorem (for the definitions of Pelczynski's properties $(V)$ and (u) we refer to [2]). 
Theorem 3. Let $X$ be a $\mathscr{L}_{\infty}$-space with Pelczynski's property (V) and $Y$ be a Banach space with Pelczynski's property $(\mathrm{u})$, not containing $l_{1}$. If $Z$ is a Banach space not containing $c_{0}$, then any $T: X \otimes_{\varepsilon} Y \rightarrow Z$ is weakly compact.

Theorem 2 has the following corollary about a new isomorphic property recently considered by Saab and Saab in [10]: a Banach space $E$ is said to possess property (w) if any operator $T: E \rightarrow E^{*}$ is weakly compact.

Corollary 4. Let $X$ be a $\mathscr{L}_{\infty}$-space with Dieudonné property and $Y$ be a Banach space not containing $l_{1}$ such that $Y^{*}$ is weakly sequentially complete. Then $X \otimes_{\varepsilon} Y$ has property (w).

Proof. It will be enough to show that $\left(X \otimes_{\varepsilon} Y\right)^{*}$ is weakly sequentially complete, because in such a case we can apply Theorem 2 . $B^{\pi}(X, Y)=\left(X \otimes_{\varepsilon} Y\right)^{*}$ is a closed subspace of $B^{\pi}\left(X^{* *}, Y\right)=\left(X^{* *} \otimes_{\varepsilon} Y\right)^{*}$ (see [3]); since $X^{* *} \otimes_{\varepsilon} Y$ is complemented in some $C(K, Y)$ space (see the proof of Theorem 2), $B^{\pi}\left(X^{* *}, Y\right)$ is complemented in $(C(K, Y))^{*}$. But now it is well known that this last space is weakly sequentially complete (see, e.g., [10, Proposition 5]). We are done. Corollary 4 is an improvement of Proposition 5 in [10].

At the end, we recall that a Banach space $E$ has the Dunford-Pettis property if any weakly compact operator on $E$ is a Dunford-Pettis (or completely continuous) operator. We observe that any $\mathscr{L}_{\infty}$-space enjoys the Dunford-Pettis property [1]. Using the same technique employed in Theorem 2, we have that $X \otimes_{\varepsilon} Y$ has the Dunford-Pettis property whenever $X$ is a $\mathscr{L}_{\infty}$-space and $Y$ is a Banach space with the Dunford-Pettis property such that $C(X, Y)$ has the same property, for any Hausdorff compact space $K$. In particular, the result in [5] and results by Bourgain quoted in [4] imply that we can choose $Y$ with the Schur property or $Y=L^{1}(\mu)$; in this last case $X \otimes_{\varepsilon} Y$ is isomorphic to the completion of the space of Pettis integrable functions (see [3]). More generally we can show that the injective tensor product of a $\mathscr{L}_{\infty}$-space and a $\mathscr{L}_{1}$-space has the DunfordPettis property.

\section{REFERENCES}

1. J. Bourgain, New classes of $\mathscr{L}_{\infty}$-spaces, Lecture Notes in Math., vol. 889, Springer-Verlag, Berlin, Heidelberg, and New York, 1981.

2. P. Cembranos, N. J. Kalton, E. Saab, and P. Saab, Pelczynski's property $(V)$ on $C(\Omega, E)$, Math. Ann. 271 (1985), 91-97.

3. J. Diestel and J. J. Uhl, Jr., Vector measures, Math. Surveys, vol. 15, Amer. Math. Soc., Providence, RI, 1977.

4. J. Diestel, A survey of results related to the Dunford-Pettis property, Contemp. Math., vol. 2, Amer. Math. Soc., Providence, RI, 1980.

5. I. Dobrakov, On representation of linear opertors on $C_{0}(T, X)$, Czechoslovak Math. J. 21 (1971), 13-30.

6. C. Fierro Bello, On weakly compact and unconditionally converging operators in spaces of vector valued continuous functions, Revista Real Acad. Madrid 81 (1987), 693-706.

7. J. L. B. Gamlen, On a theorem of Pelczynski, Proc. Amer. Math. Soc. 44 (1974), 283-285. 
8. N. J. Kalton, E. Saab, and P. Saab, On the Dieudonné property for $C(K, E)$, Proc. Amer. Math. Soc. 96 (1986), 50-52.

9. A. Pelczynski, Banach spaces on which every unconditionally converging operator is weakly compact, Bull. Acad. Polon. Sci. 10 (1962), 641-648.

10. E. Saab and P. Saab, Applications linéaires continues sur le produit tensoriel injectif d'espaces de Banach, C. R. Acad. Sci. Paris 311 (1990), 789-792.

Department of Mathematics, University of Catania, 95125 Catania, Italy 